\title{
Ventilação mecânica não invasiva com pressão positiva
}

\author{
Coordenador: Guilherme P. P. Schettino \\ Relator: Marco Antonio Soares Reis \\ Participação: Filomena Galas, Marcelo Park, Suelene Franca, Valdelis Okamoto
}

\section{Introdução}

0 uso da ventilação não invasiva com pressão positiva (VNI) para o tratamento de pacientes com insuficiência respiratória aguda ou crônica agudizada foi, certamente, um dos maiores avanços da ventilação mecânica nas últimas duas décadas. Apesar do seu uso ser relativamente recente, o grande número de séries de casos, ensaios clínicos randomizados, meta-análises ou revisões sistemáticas, assim como conferências de consenso e diretrizes publicadas até o presente momento, tornaram a aplicação dessa técnica mais "baseada em evidências" do que provavelmente qualquer outra medida de suporte ventilatório. ${ }^{(1,2)}$ Hoje não há dúvidas de que o uso da $\mathrm{VNI}$ em grupos selecionados de pacientes, como, por exemplo, pacientes com exacerbação de doença pulmonar obstrutiva crônica (DPOC), é responsável pela diminuição da necessidade de intubação, mortalidade e custos do tratamento, motivo pelo qual o seu uso vem se tornando cada vez mais freqüente.

Discutiremos a seguir as melhores evidências e recomendações para o uso da VNI em ambiente hospitalar no tratamento de pacientes adultos com insuficiência respiratória aguda ou insuficiência respiratória crônica agudizada.

\section{Aspectos técnicos para uso da ventilação não invasiva}

\section{Interfaces}

As máscaras nasais ou oronasais são as interfaces mais freqüentemente utilizadas para a aplicação da VN1 no ambiente hospitalar. A máscara nasal é, provavelmente, a interface mais confortável, porém a resistência das narinas ao fluxo de ar e a presença do vazamento de ar pela boca podem limitar o seu uso em alguns pacientes. ${ }^{(3-5)} \mathrm{A}$ máscara oronasal, também conhecida como facial, é a interface mais utilizada para pacientes com insuficiência respiratória aguda, permitindo maior volume corrente quando comparada com a máscara nasal e, conseqüentemente, correção mais rápida das trocas gasosas. ${ }^{(6)}$ Apesar dessas vantagens teóricas, não existe evidência suficiente para recomendar o uso da máscara oronasal ao invés da nasal para pacientes com insuficiência respiratória aguda. ${ }^{(7)}$ Máscaras com orifício de exalação na própria máscara podem diminuir a reinalação de $\mathrm{CO}_{2}$ quando comparadas com o uso de orifícios de exalação no circuito único dos ventiladores de VN1. ${ }^{(8)}$

$\mathrm{Na}$ tentativa de melhorar o conforto e a tolerância dos pacientes durante a VNI, dispomos hoje de novas interfaces, como, por exemplo, a máscara facial total e o capacete. A máscara facial total tem a vantagem de diminuir o vazamento e possibilitar o uso de maiores pressões inspiratórias. Uma maior área de contato entre a máscara e a face do paciente pode diminuir as lesões de pele relacionadas ao uso da máscara e tornar o seu uso mais confortável..$^{(9)}$ Schettino e colaboradores ${ }^{(8)}$ demonstraram que apesar do maior volume interno de ar nessa máscara, a reinalação de $\mathrm{CO}_{2}$ durante o uso da máscara facial total é semelhante à das máscaras oronasais. Os capacetes têm a vantagem de eliminar o contato da interface com a face do paciente, evitando assim a complicação mais freqüente da VNI, que é a lesão de pele. 0 grande espaço-morto dos capacetes e a sua parede muito complacente levam, respectivamente, à reinalação de $\mathrm{CO}_{2}$ e à necessidade do uso de maiores valores de pressão inspiratória para garantir a correção das trocas gasosas. ${ }^{(10)} 0$ ruído interno dos capacetes pode ser um grande limitante para o seu uso. ${ }^{(11)}$

\section{Ventiladores e modos ventilatórios}

Teoricamente, qualquer ventilador mecânico e modo ventilatório podem ser utilizados para a ventilação não invasiva, desde que o seu funcionamento não seja prejudicado pela presença de vazamento.

Os ventiladores específicos para VNI têm como característica principal a presença de um circuito único, por onde ocorrem tanto a inspiração como a expiração. Um orifício localizado na porção distal desse circuito é obrigatório para minimizar a reinalacão de $\mathrm{CO}_{2}$ durante a inspiração..$^{(8,12,13)}$ Esse orifício faz com que haja um vazamento contínuo de ar pelo circuito, eliminando o $\mathrm{CO}_{2}$ exalado pelo paciente durante a expiração. Por esse motivo, os ventiladores específicos para $\mathrm{VNl}$ foram desenhados para funcionar na presença de vazamento. Tolerância ao vazamento, boa 
sincronia paciente-ventilador e preço competitivo são as principais vantagens desses ventiladores, quando comparados aos ventiladores de UTI. ${ }^{(14-16)}$ Restrição de alguns modos ventilatórios, limitação de alarmes e dificuldade para o ajuste da $\mathrm{FlO}_{2}$ são algumas das limitações desses aparelhos específicos para VNI, porém alguns modelos já apresentam esses requisitos. Os novos ventiladores de UTl estão sendo adaptados para funcionarem tanto durante a ventilação invasiva quanto não invasiva. Algoritmos para a compensação automática de vazamento e a possibilidade do ajuste do critério de ciclagem da fase inspiratória para a expiração durante a pressão de suporte são exemplos de mudanças para facilitar o uso dos ventiladores de UTI para o suporte ventilatório não invasivo. Entretanto, não existe, até o momento, nenhum estudo comparando o uso de ventiladores de UTl com aqueles específicos para VNI para o suporte ventilatório não invasivo em pacientes com insuficiência respiratória aguda.

Existem relatos do uso bem sucedido de $\mathrm{VNI}$ com emprego dos modos CPAP, volume controlado, pressão controlada, pressão de suporte e ventilação assistida proporcional (PAV).

Para pacientes com DPOC agudizada, pressão de suporte e PEEP (PS + PEEP ou inspiratory positive airway pressure, também conhecido como IPAP, nível acima da EPAP - expiratory positive airway pressure) foi o modo ventilatório utilizado na maioria dos estudos publicados até o momento, sendo por isto o modo ventilatório recomendado pela maioria dos autores. ${ }^{(17)}$ Nessa modalidade, a PS (ou IPAP) deve ser ajustada para gerar um volume corrente por volta de 6 a $8 \mathrm{~mL} / \mathrm{kg}$ e freqüência respiratória $<30 / \mathrm{min}$. Valor da PEEP (ou EPAP) deve ser inferior ao da PEEP intrínseca (devido à dificuldade de medir a PEEP intrínseca durante a ventilação espontânea, sugere-se o uso da PEEP/EPAP inicialmente em torno de $6 \mathrm{cmH}_{2} 0$ ). A ventilação assistida proporcional (PAV) pode ser uma alternativa a PS + PEEP para VNI na DPOC agudizada, como demonstrado em alguns estudos clínicos. ${ }^{(18-20)}$

CPAP (continuous positive airway pressure) é um modo ventilatório empregado com frequência para o suporte ventilatório não invasivo de pacientes com edema agudo de pulmão (EAP). Nessa situação, acreditamos que se deva utilizar um valor mínimo de $10 \mathrm{cmH}_{2} \mathrm{O}$ para garantir os benefícios hemodinâmicos e ventilatórios da CPAP. Lembremos que a CPAP não é capaz de aumentar a ventilação alve- olar, motivo pelo qual, na presença de hipercapnia, é dada preferência ao uso da ventilação não invasiva com dois níveis de pressão. ${ }^{(21,22)} \mathrm{Fu}$ et al. ${ }^{(23)}$ demonstraram que a CPAP obtida com gerador de fluxo tem funcionamento semelhante à CPAP de ventiladores mecânicos para aplicação não invasiva.

\section{Limitações para o uso da ventilação não invasiva}

A adequada seleção dos candidatos ao uso da ventilação não invasiva é o primeiro passo para o sucesso da técnica (Quadro 1). Como a VNl é uma modalidade de suporte ventilatório parcial e sujeita a interrupções, essa técnica não deve ser utilizada em pacientes totalmente dependentes da ventilação mecânica para se manterem vivos. A cooperação do paciente é importante para o sucesso da VNl, tornando o seu uso limitado nos pacientes com rebaixamento do estado de consciência ou com agitação. Da mesma forma, pela inexistência de uma prótese traqueal a VNI só deve ser utilizada naqueles pacientes capazes de manter a permeabilidade da via aérea superior, assim como a integridade dos mecanismos de deglutição e a capacidade de mobilizar secreções. Instabilidade hemodinâmica grave, caracterizada pelo uso de aminas vasopressoras, e arritmias complexas são consideradas contra-indicações para o uso da VNI pela maioria dos autores. Pacientes com distensão abdominal

Quadro 1 - Contra-indicações para o uso da ventilação não invasiva com pressão positiva.

Diminuição da consciência, sonolência, agitação, confusão ou recusa do paciente Instabilidade hemodinâmica com necessidade de medicamento vasopressor, choque (pressão arterial sistólica $<90 \mathrm{mmHg}$ ), arritmias complexas

Obstrução de via aérea superior ou trauma de face

Tosse ineficaz ou incapacidade de deglutição Distensão abdominal, náuseas ou vômitos

Sangramento digestivo alto

Infarto agudo do miocárdio

Pós-operatório recente de cirurgia de face, via aérea superior ou esôfago

Uso de VN1 é controverso: pós-operatório de cirurgia gástrica, gravidez 
ou vômitos não devem utilizar VN1 pelo risco de aspiração. Pós-operatório imediato de cirurgia do esôfago é contra-indicação para VNI, entretanto dúvidas persistem acerca da segurança do seu uso no pós-operatório de cirurgias gástricas. Trauma de face, lesão aguda e/ou sangramento de via aérea são também consideradas limitações para o uso da VN1.

\section{Indicações para o uso da ventilação não invasiva}

Descreveremos, a seguir, as recomendações e os graus de evidência para o emprego da VNI nas condições mais freqüentes para o seu uso em ambiente hospitalar, com enfoque na redução da taxa de intubação e da mortalidade. Caso existam estudos com diferentes níveis de evidência e concordantes em recomendar ou não o uso da $\mathrm{VNl}$, nos limitaremos a descrever somente aqueles estudos com os maiores níveis de evidências.

\section{Ventilação não invasiva na exacerbação da doença pulmonar obstrutiva crônica}

Recomendação: VN1 deve ser utilizada como tratamento de primeira escolha para pacientes com agudização da DPOC, especialmente para aqueles pacientes com exacerbação grave, caracterizada pela presença de acidose respiratória $(\mathrm{pH}<7,35)$ que persiste a despeito de tratamento médico máximo associado a oxigenoterapia controlada. 0 uso de VNI diminui a necessidade de intubação e reduz a mortalidade hospitalar desses pacientes. Por esses motivos, essa intervenção deve estar disponível nos hospitais que atendam pacientes com exacerbação de DPOC.

Grau de recomendação: A

Comentário: A evidência mais forte para o benefício do uso da VNI, tanto para a diminuição da necessidade de intubação quanto para a redução da mortalidade, é no tratamento da exacerbação da DPOC. Existem, até o momento, três metaanálises ${ }^{(24-26)}$ baseadas em estudos controlados e randomizados, realizados exclusivamente em pacientes com DPOC agudizada ${ }^{(27-34)}$ que comprovam o benefício do uso da VN1 para diminuir tanto a necessidade de intubação quanto a mortalidade hospitalar, quando a ventilação não invasiva é comparada com o tratamento convencional para esses pacientes. Entretanto, a análise de Keenan et al. ${ }^{(25)}$ reportou que esses benefícios não foram demonstrados em pacientes com exacerbações mais leves de DPOC, enfatizando o conceito de que a VNI é indicada para pacientes com exacerbações mais graves, acompanhadas de hipercapnia e acidose respiratória. Esse conceito foi recentemente questionado por um estudo randomizado chinês, com 342 pacientes, em que a VNI foi aplicada a pacientes em fase precoce (entre 24 e $48 \mathrm{~h}$ ) de exacerbação aguda de DPOC que demonstrou benefício também para os casos com menor alteração de $\mathrm{pH}$ e $\mathrm{PaCO}_{2}$. Nesse estudo, houve menor taxa de intubação no grupo que recebeu VN1 (4,7\% vs. 15,2\% no grupo controle, $p=0,002)$. A diferença de mortalidade entre os grupos, porém, não foi significativa $(4,1 \%$ vs. $7,0 \%)^{(35)}$

A Tabela 1, adaptada de Hess ${ }^{(17)}$, descreve o benefício da $\mathrm{VNl}$ em diminuir a necessidade de intubação

Tabela 1 - Ventilação não invasiva para exacerbação da DPOC: Meta-Análises.

\begin{tabular}{|c|c|c|c|}
\hline Autor/Ano & Estudos (n) & Intubação & Mortalidade \\
\hline Lightowler, 2003 & 8 & $\begin{array}{l}\text { Risco Relativo }=0,42 \\
1 \mathrm{C} 95 \%=0,31 \text { a } 0,59\end{array}$ & $\begin{array}{l}\text { Risco Relativo }=0,41 \\
1 \mathrm{C} 95 \%=0,26 \text { a } 0,64\end{array}$ \\
\hline Keenan, 2003 & 15 & $\begin{array}{l}\text { Exacerbação grave*: } \\
\text { Redução do Risco = 34\% } \\
1 \text { 1C95\% = 22\% a } 46 \% \\
\text { Exacerbação não grave: } \\
\text { Redução do Risco = 0\% } \\
\text { 1C95\% = -11\% a 11\% }\end{array}$ & $\begin{array}{l}\text { Exacerbação grave*: } \\
\text { Redução do Risco }=12 \% \\
1 \text { C95\% } \% 6 \% \text { a } 18 \% \\
\text { Exacerbação não grave: } \\
\text { Redução do Risco }=2 \% \\
1 \text { C95\% }=-8 \% \text { a } 12 \%\end{array}$ \\
\hline Ram, 2004 & 14 & $\begin{array}{l}\text { Risco Relativo }=0,41 \\
1 C 95 \%=0,33 \text { a } 0,53\end{array}$ & $\begin{array}{l}\text { Risco Relativo }=0,52 \\
1 \mathrm{C} 95 \%=0,35 \text { a } 0,76\end{array}$ \\
\hline
\end{tabular}

n: número de estudos incluídos na meta - análise; 1C: intervalo de confiança; *Exacerbação grave: definida como acidose respiratória ( $\mathrm{pH}<7,30$ ) ou mortalidade hospitalar observada no grupo controle $>10 \%$. Observação: Risco relativo <1 indica proteção. 
e mortalidade para pacientes com exacerbação da DPOC segundo as referidas meta-análises.

\section{Ventilação não invasiva na exacerbação da asma}

Recomendação: VNI pode ser utilizada em conjunto com o tratamento medicamentoso convencional para o cuidado de pacientes selecionados com exacerbação aguda e grave da asma.

Grau de Recomendação: B

Comentário: 0 sucesso do emprego da $\mathrm{VNI}$ no tratamento da agudização da DPOC fez com que o seu uso fosse também tentado no tratamento da exacerbação da asma. Meduri e colaboradores ${ }^{(36)}$ descreveram o uso da VN1 em uma série de 17 pacientes com exacerbação aguda de asma e relataram que a intubação foi necessária em apenas três desses casos. Soroksky e colegas $^{(37)}$ realizaram um estudo randomizado em 30 pacientes com agudização de asma, que incluía o uso de uma manobra de "VNl-placebo" no grupo controle (que consistia na utilização de VNI com pressões ins e expiratória de $1 \mathrm{cmH}_{2} \mathrm{O}$ ), para avaliar o benefício da $\mathrm{VNI}$ nessa condição. Esses autores demonstraram que os pacientes que receberam VNl por $3 \mathrm{~h}$ consecutivas apresentaram melhora mais rápida dos sintomas, aumento do $\mathrm{VEF}_{1}$ e diminuição da necessidade de internação em relação ao grupo controle. Revisão sistemática recente da $\operatorname{Cochrane}^{(38)}$ sobre o tema não encontrou nenhum outro estudo relevante, a não ser o descrito acima, concluindo que o uso da VNI para o tratamento da exacerbação grave da asma parece promissor, porém o único estudo publicado e o limitado número de pacientes avaliados não permitem a recomendação definitiva para o seu uso.

\section{Ventilação não invasiva no edema pulmonar cardiogênico}

\section{CPAP no edema pulmonar cardiogênico}

Recomendação: 0 uso de CPAP é seguro e diminui a necessidade de intubação para pacientes com edema agudo de pulmão, devendo ser aplicado precocemente e em conjunto com a terapia medicamentosa convencional.

Grau de recomendação: A

Comentário: As evidências para a utilização de CPAP em insuficiência respiratória aguda vêm de três ensaios clínicos randomizados ${ }^{(39-41)}$ que compararam CPAP com tratamento convencional em pacientes com edema pulmonar cardiogênico (EPC). Rasanen e colaboradores ${ }^{(39)}$ compararam o tratamento convencional isolado ou associado ao uso de pressão positiva contínua em vias aéreas (CPAP = $10 \mathrm{cmH}_{2} \mathrm{O}$ ), para pacientes com edema pulmonar de origem cardíaca. Nesse estudo, houve apenas uma melhora mais rápida da hipoxemia no grupo que usou CPAP. No estudo de Bersten ${ }^{(40)}, 39$ pacientes com EPC e alto risco de intubação, caracterizado pela presença de hipercapnia, foram randomizados para receber terapia convencional, associada ou não a $\mathrm{CPAP}=10 \mathrm{cmH}_{2} \mathrm{O}$. Além da melhora mais rápida da hipercapnia e da hipoxemia, nenhum paciente que recebeu CPAP foi intubado, contra 35\% daqueles em tratamento convencional. $\mathrm{Na}$ casuística de lin et al. ${ }^{(41)}$, com 100 pacientes, foi aplicado CPAP em níveis progressivamente maiores, variando de 2,5 até $12,5 \mathrm{cmH}_{2} 0$, num intervalo de $2 \mathrm{~h}$ e meia. 0 resultado foi concordante com o estudo de Bersten $^{(40)}$. A análise conjunta desses três estudos ${ }^{(42)}$ mostrou uma redução na necessidade de intubação (diferença de risco $=-26 \%, 1 C 95 \%=-13 \%$ a $-38 \%$ ) e uma tendência à redução na mortalidade (diferença de risco $=-6,6 \%, 1 \mathrm{C} 95 \%=3 \%$ a $-16 \%$ ) com o uso do CPAP. Em um estudo mais recente, L'Her e colaboradores $^{(43)}$ demonstraram, em 89 pacientes consecutivos, com idade acima de 80 anos, a superioridade do uso de CPAP $=10 \mathrm{cmH}_{2} \mathrm{O}$ em termos de redução de complicações (17 pacientes no grupo controle vs. 4 pacientes no grupo CPAP; $p=0,002$ ) e de mortalidade com $48 \mathrm{~h}$ de inclusão (tratamento convencional $=24 \%$ vs. $\mathrm{CPAP}=7 \% ; \mathrm{p}=0,017)$. Porém, não houve diferença nas taxas de mortalidade hospitalar entre os dois grupos de estudo.

\section{VNI com dois níveis de pressão no edema pulmonar cardiogênico}

Recomendação: Pacientes com hipercapnia associada à hipoxemia parecem ser os que mais se beneficiam do uso da VNl com PEEP acrescido de pressão de suporte (PEEP + PS) no tratamento do EPC. 0 uso de pressão expiratória de $10 \mathrm{cmH}_{2} \mathrm{O}$ parece ser o ponto chave do benefício respiratório/ hemodinâmico para pacientes com edema agudo dos pulmões de origem cardíaca, tanto durante o uso do CPAP, quanto na VNI com PEEP + PS.

Grau de recomendação: B 
Comentário:

A ) VNl com PEEP + PS vs. CPAP: Não é claro, no momento, se VNI com PEEP + PS é superior a CPAP no tratamento do EPC. ${ }^{(44,45)}$ No primeiro estudo utilizando VNI com PEEP + PS para o tratamento do EPC, Mehta e colaboradores ${ }^{(46)}$ compararam $^{2}$ 14 pacientes em uso de $\mathrm{VNI}$ com PS $=15 \mathrm{cmH}_{2} \mathrm{O}+$ PEEP $=5 \mathrm{cmH}_{2} \mathrm{O}$, com 13 pacientes em uso de $\mathrm{CPAP}=10 \mathrm{cmH}_{2} \mathrm{O}$. Houve, na análise intragrupo, uma queda mais rápida na pressão arterial, freqüência cardíaca e respiratória no grupo randomizado para o uso de VNI com PEEP + PS. A taxa de intubação foi semelhante, porém o resultado que tornou esse estudo polêmico foi a elevada taxa de infarto agudo do miocárdio no grupo VN1 com PEEP + PS (71\% vs. 31\%), o que resultou na interrupção do estudo. No ensaio clínico de Bellone et al. ${ }^{(47)}, 36$ pacientes com edema agudo dos pulmões e hipercapnia $\left(\mathrm{PaCO}_{2}>45 \mathrm{mmHg}\right)$ foram randomizados para tratamento com CPAP $=10 \mathrm{cmH}_{2} \mathrm{O}$ ou VNI com PEEP $\left.=5 \mathrm{cmH}_{2} \mathrm{O}+\mathrm{PS}=15 \mathrm{cmH}_{2} \mathrm{O}\right)$. Nesse estudo, não houve diferença entre os grupos com relação a tempo para melhora clínica, taxa de intubação e mortalidade hospitalar. Níveis de troponina 1 sérica e de infarto agudo do miocárdio foram semelhantes nos dois grupos.

B) VN1 COM PEEP + PS vs. oxigenoterapia: Também não é claro, no momento, se VNI com PEEP + PS é superior ao tratamento convencional do EPC (o benefício parece existir em pacientes com hipercapnia). No estudo randomizado de Masip e colaboradores $^{(21)}$, comparando a terapia convencional, associada ou não ao uso da VNI com PEEP + PS, houve melhora da hipoxemia, redução da taxa de intubação (5\% vs. 33\%) e recuperação mais rápida da hipercapnia no grupo randomizado para VN1 com PEEP + PS. Os efeitos benéficos do uso da VN1 com PEEP + PS foram mais evidentes para aqueles pacientes hipercápnicos na randomização. A incidência de infarto agudo do miocárdio foi semelhante em ambos os grupos. Nava e colaboradores $^{(48)}$ estudaram 130 pacientes que receberam VNI com PEEP $=5 \mathrm{cmH}_{2} \mathrm{O}+\mathrm{PS}=15 \mathrm{cmH}_{2} \mathrm{O}$ ou terapia convencional. Apenas os pacientes com hipercapnia $\left(\mathrm{PaCO}_{2}>45 \mathrm{mmHg}\right)$ alocados para uso da $\mathrm{VNI}$ com PEEP + PS tiveram redução na taxa de intubação ( $6 \%$ vs. $28 \%, p=0,015)$. A taxa de infarto agudo do miocárdio foi igual nos dois grupos.

C) VNI com peep + PS vs. cpap vs. oxigenoterapia: No estudo de Crane ${ }^{(49)}, 60$ pacientes foram randomizados para tratamento convencional, $\mathrm{CPAP}=10 \mathrm{cmH}_{2} \mathrm{O}$ ou VNl com PEEP $=5 \mathrm{cmH}_{2} \mathrm{O}+$ $\mathrm{PS}=15 \mathrm{cmH}_{2} \mathrm{O}$. Houve melhora semelhante entre os grupos na oxigenação, acidose e queda da freqüência respiratória. As taxas de intubação e de infarto agudo do miocárdio também foram semelhantes entre os grupos. No entanto, a mortalidade hospitalar maior nos grupos controle e VNI com PEEP + PS, comparados com o grupo CPAP (30\% no grupo controle, $15 \%$ no grupo VNl com PEEP + PS e 0\% no grupo CPAP). No estudo de Park e associados $^{(22)}, 80$ pacientes com EPC foram randomizados para receber tratamento convencional (oxigenoterapia com $\mathrm{FlO}_{2}=0,5$ ), $\mathrm{CPAP}=10 \mathrm{cmH}_{2} \mathrm{O}$ ou VNI com PS $=15 \mathrm{cmH}_{2} \mathrm{O}+\mathrm{PEEP}=10 \mathrm{~cm} \mathrm{H}_{2} \mathrm{O}$. Além da melhora equivalente dos sinais vitais nos grupos CPAP e VNI com PEEP + PS, observaram uma melhora nitidamente superior emm relação ao grupo tratado de forma convencional, a taxa de intubação foi reduzida de $42 \%$ durante a terapia convencional para 7\% em ambos os grupos que usaram pressão positiva não invasiva, havendo também redução da mortalidade em 14 dias a favor dos pacientes que utilizaram pressão positiva não invasiva (CPAP e VN1 com PEEP + PS). Não houve aumento da incidência de infarto agudo do miocárdio nessa população.

\section{Ventilação não invasiva na insuficiência respiratória hipoxêmica}

Recomendação: A VN1 pode ser benéfica na insuficiência respiratória hipoxêmica, porém seu uso deve ser cauteloso.

Grau de recomendação: B

Comentário: A insuficiência respiratória hipoxêmica pode ser causada por várias doenças, com características fisiopatológicas e curso clínico distintos, o que torna a avaliação dos benefícios do uso da VN1 para o tratamento de pacientes nessa condição complexa e controversa. ${ }^{(1,17,50-53)}$ Alguns estudos, apesar de considerarem casuísticas heterogêneas, mostram benefícios do uso da VNI em reduzir a necessidade de intubação, complicações associadas à ventilação mecânica e mesmo mortalidade quando a VN1 é comparada com tratamento convencional, ou mesmo com a ventilação invasiva, para o cuidado de pacientes com insuficiência respiratória hipoxêmica. Em um dos primeiros ensaios clínicos de VNI na insuficiência respiratória hipoxêmica, Antonelli et al.(54), em 1998, compararam 
64 pacientes com insuficiência respiratória aguda de diversas etiologias que foram submetidos à ventilação mecânica invasiva ou VN1. 0 uso da VNI foi tão efetivo quanto a ventilação convencional para a correção da hipoxemia, mas o grupo $\mathrm{VNI}$ apresentou menor tempo de ventilação mecânica e alta mais precoce da UTl, além de apresentar menos complicações associadas à ventilação mecânica. Ferrer et al. ${ }^{(5)}$ publicaram estudo realizado em três centros na Espanha, no qual se comparou o uso da VNI com o tratamento convencional baseado no uso de máscara Venturi com altas $\mathrm{FlO}_{2}$ em 105 pacientes com insuficiência respiratória aguda grave de diversas etiologias. Observaram que a $\mathrm{VNI}$ se associou à menor necessidade de intubação (13,25\% vs. 28,52\%), menor incidência de choque séptico $(6,12 \%$ vs. $17,31 \%)$, menor mortalidade na UTI (9,18\% vs. 21,39\%), além de maior sobrevida em 90 dias.

Já existem três revisões sistemáticas ou metanálises sobre o uso da VNI na insuficiência respiratória hipoxêmica ${ }^{(56-58)}$ (Tabela 2). Na mais recente delas, Keenan e associados ${ }^{(58)}$ avaliaram os estudos randomizados publicados até outubro de 2003 sobre o uso da $\mathrm{VNl}$ em pacientes com insuficiência respiratória aguda hipoxêmica não relacionada ao edema agudo de pulmão. Os resultados dessa revisão sistemática comprovam que o uso da VNI está associado à redução na necessidade de intubação (redução de risco $=24 \%$ [1C95\%: $8 \%$ a 36\%]), redução nos dias de permanência na UTl em 1,9 dia (1C95\%: 1,0 a 2,9 dias), redução da mortalidade na UT1 16\% (1C95\%: $5 \%$ a $27 \%$ ) e a uma tendência à redução da mortalidade hospitalar de 10\% (1C95\%: -7\% a -27\%). Os autores dessa revisão sistemática salientam, entretanto, que os resultados apresentados sejam avaliados com cuidado, pois é possível que, dentro da população heterogênea de pacientes com insuficiência respiratória aguda, existam subgrupos que se beneficiem com a $\mathrm{VNl}$, como, por exemplo, pacientes imunossuprimidos ou pós-operatório de toracotomia e outros, nos quais o seu efeito benéfico ainda não foi comprovado. Aconselham ainda que novos estudos randomizados sejam realizados em grupos específicos de pacientes com insuficiência respiratória aguda hipoxêmica.

\section{Ventilação não invasiva na insuficiência respiratória hipoxêmica em condições especificas}

Recomendação: 0 uso da VNI parece ser útil para diminuir a mortalidade em subgrupos específicos de pacientes com insuficiência respiratória hipoxêmica, como, por exemplo, pacientes imunossuprimidos.

Grau de Recomendação: B

Comentário:

A) Imunossupressão e pós-transplante: A morbimortalidade da pneumonia associada à ventilação mecânica invasiva é alta para pacientes imunossuprimidos e transplantados de medula óssea ou órgãos sólidos. Antonelli e colaboradores ${ }^{(59)}$, em 2000, publicaram estudo no qual avaliaram, de forma aleatorizada e controlada, a aplicação da VNI, comparando-a com o tratamento padrão, incluindo suplementação de oxigênio administrado por máscara facial, em 40 receptores de órgãos sólidos, que desenvolveram insuficiência respiratória aguda hipoxêmica. Observaram melhora precoce e mantida da oxigenação e menor necessidade de intubação no grupo VN1 comparado com o tratamento padrão (20\% vs. $70 \%)$. Também houve menor mortalidade no grupo que recebeu VNI (20\% vs. 50\%). Hilbert et al. ${ }^{(60)}$ compararam a aplicação precoce e intermitente de VNI com o tratamento convencional, incluindo a suplementação de oxigênio, em 52 pacientes imunossuprimidos, a maioria deles transplantados de medula óssea, com insuficiência respiratória aguda, febre e infiltrados pulmonares. No grupo submetido à VNI houve

Tabela 2 - VNI para insuficiência respiratória aguda hipoxêmica: Meta-Análises.

\begin{tabular}{ccll}
\hline Autor/Ano & Estudos $(\mathrm{n})$ & \multicolumn{1}{c}{ Intubação } & \multicolumn{1}{c}{ Mortalidade } \\
\hline Keenan, 1997 & 7 & $\begin{array}{l}\text { Odds ratio }=0,20 \\
\text { IC95\% }=0,11 \text { a } 0,36\end{array}$ & Odds ratio $=0,29$ \\
IC95\% $=0,15$ a 0,59 \\
Peter, 2002 & 15 & Diferença de risco $=-0,19$ & Diferença de risco $=-0,08$ \\
& & IC95\% $=-0,28$ a $-0,09$ & 1C95\% $=-0,16$ a $-0,01$ \\
Keenan, 2004 & \multirow{2}{*}{14} & Risco Relativo $=0,41$ & Risco Relativo $=0,52$ \\
& & IC95\% $=0,33$ a 0,53 & IC95\% $=0,35$ a 0,76 \\
\hline
\end{tabular}

n: número de estudos incluídos na meta-análise; lC: intervalo de confiança. 
menor necessidade de intubação (12\% vs. 20\%), menor número de complicações graves $(13 \%$ vS. $21 \%$ ), o que levou à menor mortalidade na UT1 (10\% vs. 18\%) e hospitalar (13\% vs. 21\%). Também na insuficiência respiratória aguda relacionada à pneumonia por Pneumocystis carinii, em pacientes com AIDS, parece haver benefício do uso da VN1. Confalonieri e associados ${ }^{(61)}$, em estudo prospectivo, tipo caso-controle, publicado em 2002, no qual 24 pacientes tratados com $\mathrm{VNI}$ foram comparados com 24 pacientes tratados com ventilação mecânica invasiva demonstraram que o uso de VN1 evitou a intubação em 67\% dos pacientes tratados, o que se associou com melhor sobrevida na UT1 (100\% vs. 38\%; $p=0,003)$, no hospital e aos 60 dias da inclusão.

B) Pneumonia: Confalonieri et al.(62) estudaram a aplicação de VNI comparada ao tratamento padrão, de forma randomizada e controlada, em 56 pacientes com pneumonia comunitária grave e relacionaram a utilização de VNI com menor necessidade de intubação $(21 \%$ vs. $50 \%, p=0,03)$ e menor tempo de permanência na UTI ( 1,8 dia vs. 6 dias, $p=0,04)$. Os dois grupos tiveram a mesma mortalidade hospitalar, entretanto, entre os pacientes com DPOC, aqueles randomizados para VN1 apresentaram uma maior sobrevida aos dois meses $(88,9 \%$ vs. $37,5 \%$, $\mathrm{p}=0,005)$.

C) Pós-ressecção pulmonar: Auriant e colaboradores $^{(63)}$ avaliaram 47 pacientes que desenvolveram IRA após cirurgia de ressecção pulmonar, randomizados para $\mathrm{VNI}$ ou tratamento convencional, relatando menor necessidade de intubação endotraqueal $(21 \%$ vs. $50 \%)$ e menor taxa de mortalidade $(12,5 \%$ vs. $37,5 \%)$ nos pacientes submetidos à VN1.

D) Lesão pulmonar aguda/Síndrome do desconforto respiratório agudo (LPA/SDRA): Rocker e associados $^{(64)}$ estudaram uma série de 12 episódios de LPA/SDRA, nos quais foi empregada a VNI, por máscara facial, utilizando dois níveis pressóricos. A taxa de sucesso foi de 50\% e a sobrevida, de $70 \%$. No estudo de Ferrer et al.(55), no qual se comparou a aplicação de $\mathrm{VNI}$ com altas $\mathrm{FlO}_{2}$ administradas por máscara Venturi, em pacientes com insuficiência respiratória aguda grave, havia 15 pacientes (14,5\% do total de 105 pacientes) com LPA/SDRA. Desses, sete foram randomizados para VN1 e oito tratados no grupo controle. Apesar dos resultados gerais do estudo terem sido favoráveis à utilização da VNI, os desfechos nos pacientes com LPA/SDRA foram muito ruins. A taxa de intubação foi alta nos pacientes com LPA/SDRA em ambos os grupos (86\% no grupo VN1 vs. $100 \%$ no grupo $\mathrm{O}_{2}$ ), assim como a mortalidade (71\% vs. $87,5 \%$ respectivamente).

\section{Cuidados especiais durante a ventilação não invasiva na insuficiência respiratória hipoxêmica}

Recomendação: Devido ao alto risco de falência da VNI e conseqüente necessidade de intubação, pacientes com insuficiência respiratória hipoxêmica devem receber VN1 onde existam facilidades para vigilância, monitoração, intubação traqueal e ventilação invasiva.

Grau de recomendação: D

Comentário: Entendemos que a VNI pode ser tentada em pacientes com insuficiência respiratória hipoxêmica desde que o paciente não apresente contra-indicações para o seu uso. No entanto, defendemos que seu uso deva ser feito em ambiente com supervisão médica constante, com possibilidade de monitoração dos sinais vitais, incluindo oximetria de pulso e com facilidades para intubação. Pacientes que necessitem de $\mathrm{FlO}_{2}>0,6$, aqueles que apresentem queda rápida da oxigenação $\left(\mathrm{SaO}_{2}<90 \%\right)$ após a retirada da máscara e pacientes que não apresentem melhora do seu quadro nas primeiras $2 \mathrm{~h}$ de uso da $\mathrm{VNl}$ devem ser considerados para intubação eletiva e segura.

\section{Ventilação não invasiva em pacientes terminais}

Recomendação: VNl pode ser usada em pacientes terminais quando a causa da insuficiência respiratória for potencialmente reversível, particularmente naqueles pacientes com DPOC agudizada ou com edema pulmonar de origem cardíaca.

Grau de recomendação: B

Comentário: 0 uso da VNI pode ser útil para tratar a insuficiência respiratória de pacientes terminais, desde que a causa da insuficiência respiratória seja potencialmente reversível e não represente somente a evolução final de doença pulmonar ou extrapulmonar. Vale salientar que alguns pacientes com doenças terminais referem o desejo de não serem intubados, mas aceitam o uso da ventilação não invasiva como forma de suporte ventilatório para o tratamento da insuficiência respiratória ou mesmo como medida paliativa para alívio da dispnéia. As 
evidências para o uso da $\mathrm{VNl}$ em pacientes terminais são escassas e baseiam-se em estudos observacionais. Meduri e colaboradores ${ }^{(65)}$ descreveram sobrevida hospitalar em $64 \%$ de 11 pacientes com DPOC agudizada, que haviam previamente recusado intubação, e que receberam VN1 para tratamento da exacerbação da doença pulmonar. Benhamou e associados ${ }^{(66)}$ publicaram resultados semelhantes quando VN1 foi utilizado em 30 pacientes, a maioria deles com insuficiência respiratória hipercápnica, que haviam recusado intubação ou que esse procedimento foi considerado inapropriado, seja pela idade ou presença de co-morbidades, com taxa de sucesso de 60\%. O benefício do uso da VNI para pacientes com DPOC avançado foi também sugerido por Chu et al.(67) $\mathrm{em}$ um estudo com controle histórico. Em estudo observacional recente, Levy e colaboradores $^{(68)}$ descreveram que de um total de 1.211 pacientes consecutivos que usaram VNI para tratamento de insuficiência respiratória aguda em quatro centros americanos, 114 haviam declarado o desejo de não serem intubados. A sobrevida hospitalar destes pacientes foi de 57\%, sendo a presença de hipercapnia e diagnóstico de insuficiência cardíaca associada a uma melhor evolução, enquanto que a presença de pneumonia ou de câncer determinava pior prognóstico. Schettino et al.(69) desmostraram que o uso de $\mathrm{VNl}$ em pacientes que não seriam intubados pode ser benéfico para aqueles pacientes com o diagnóstico de DPOC agudizado e insuficiência cardíaca congestiva, mas não em pacientes com insuficiência respiratória hipoxêmica, insuficiência respiratória pós-extubação ou pacientes com doença oncológica avançada. Um escore SAPS II > 35 pontos e albumina $<2,5 \mathrm{~g} / \mathrm{dL}$ foram as variavéis independentes associadas à mortalidade hospitalar desses pacientes.

\section{Ventilação não invasiva no periodo pós- operatório imediato}

Recomendação: A VNI pode ser utilizada com cautela para o tratamento da insuficiência respiratória hipoxêmica no período pós-operatório imediato de cirurgias abdominais e torácicas eletivas. A utilização da VN1 no período pós-operatório deve respeitar as limitações e contra-indicações para o seu uso.

Grau de recomendação: B
Comentário: 0 uso da VN1 para tratamento de insuficiência respiratória hipoxêmica no período pósoperatório imediato de cirurgia abdominal e torácica eletivas está associado à diminuição da necessidade de intubação e possivelmente diminuição da mortalidade. VNI administrada através de máscara ou peças bucais, de forma contínua ou intermitente, tem sido utilizada isoladamente ou associada a manobras fisioterápicas para a prevenção de atelectasia e hipoxemia no período pósoperatório de cirurgias abdomimais, porém com resultados conflitantes ${ }^{(70-74)}$. Existem descrições também do uso de VNI no pós-operatório de cirurgia bariátrica, seja na tentativa de evitar atelectasia ou para tratamento de hipoventilação associada à apnéia obstrutiva do sono, tão freqüente nesses pacientes ${ }^{(75)}$. Joris e colaboradores ${ }^{(76)}$ demonstraram, em um estudo randomizado, que o uso de $\mathrm{PS}=12 \mathrm{cmH}_{2} \mathrm{O}+\mathrm{PEEP}=4 \mathrm{cmH}_{2} \mathrm{O}$ nas primeiras $24 \mathrm{~h}$ de pós-operatório de gastroplastia melhorou a função respiratória de pacientes obesos quando comparado com o tratamento convencional ou PS $=8 \mathrm{cmH}_{2} \mathrm{O}+$ PEEP $=4 \mathrm{cmH}_{2} \mathrm{O} .0$ estudo randomizado e multicêntrico de Squadrone e colaboradores demonstrou pela primeira vez que VNI (CPAP de 7,5 $\mathrm{cmH}_{2} \mathrm{O}$ administrado através de capacete), quando utilizada de forma precoce para o tratamento de hipoxemia $\left(\mathrm{PaO}_{2} / \mathrm{FlO}_{2}<\right.$ $300 \mathrm{mmHg}$ ) no pós-operatório imediato de cirurgia abdominal eletiva e de grande porte, incluindo gastrectomias, leva à redução da necessidade de intubação traqueal e de pneumonia nosocomial, à diminuição do tempo de internação na unidade de terapia intensiva, sem resultar em aumento de complicações associadas à cirurgia ${ }^{(77)}$.

Pacientes no pós-operatório de cirurgia torácica também podem se beneficiar do uso de VNl. Aguilo e colaboradores demonstraram, em um estudo randomizado, que o uso de PS + PEEP por máscara nasal foi útil para corrigir as trocas gasosas quando comparado com tratamento convencional em pacientes no pós-operatório de toracotomia para ressecção pulmonar, sem, contudo, levar a complicações pósoperatórias como, por exemplo, fístula pleural ${ }^{(78)}$. Auriant et al., também em um estudo randomizado, demostraram que o uso de VN1 para o tratamento de insuficiência respiratória hipoxêmica no pósoperatório de ressecção pulmonar está associado à menor necessidade de intubação (20,8 vs. 50\%) e mortalidade (12,5 vs. 37,5\%), quando comparado com o tratamento convencional ${ }^{(63)}$. Vários autores demostraram que o uso de CPAP, ou preferencial- 
mente PS + PEEP, é seguro e pode ser útil para melhorar a função pulmonar e as trocas gasosas no pós-operatório de cirurgia de revascularização do miocárdio, entretanto a importância clínicas desses achados necessita de confirmação( ${ }^{(79-81)}$.

É importante ressaltar que, embora algumas das recomendações ou consensos publicadas até o momento incluam cirurgia do trato digestivo alto como contra-indicação para VNI, alguns autores defendem que essa técnica pode ser utilizada no pós-operatório de gastrectomia, desde que haja avaliação criteriosa do paciente e cuidado com a técnica de $\mathrm{VNl}$ a ser empregada. 0 uso de CPAP limitado a 7,5 $\mathrm{cmH}_{2} \mathrm{O}$ ou mesmo PS + PEEP, desde que o valor de pico de pressão inspiratória não ultrapasse $10 \mathrm{cmH}_{2} \mathrm{O}$ e a PEEP não seja maior que $5 \mathrm{cmH}_{2} \mathrm{O}$, parece ser uma maneira segura de se empregar VN1 no período pós-operatório de cirurgia abdominal e ressecção pulmonar eletivas, na tentativa de evitarem-se complicações associadas à VNI no pós-operatório, principalmente distensão gástrica e vômitos. 0 uso do capacete como interface parece ser uma opção à máscara facial para aqueles pacientes com necessidade de uso da sonda nasogátrica no período pós-operatório imediato.

Acreditamos, entretanto, que VN1 não deve ser utilizada no pós-operatório imediato de esofagectomia ou naqueles pacientes com distensão abdominal, náuseas e vômitos, deiscências, perfurações ou outras complicações operatórias do trato gastrointestinal.

\section{Ventilação não-invasiva na insuficiência respiratória pós-extubação}

Recomendação: A VN1 não deve ser utilizada como método de resgate na insuficiência respiratória desenvolvida após a extubação, pois ela pode retardar a reintubação.

Grau de recomendação: A

Comentário: Insuficiência respiratória após a extubação pode ocorrer mesmo após uma adequada condução do desmame e até o momento não há nenhum parâmetro objetivo que possa identificar os pacientes em risco. Cerca de 13\% a 19\% dos pacientes extubados necessitam de reintubação. Eles apresentam mortalidade sete vezes maior que os extubados com sucesso. Dentre as principais causas de reintubação estão a obstrução de vias aéreas superiores (edema de glote), fadiga muscular respi- ratória, edema cardiogênico, infecções, inabilidade de eliminar secreções, hipoxemia grave, alteração no estado mental e balanço hídrico positivo. Algumas séries de casos e estudos não randomizados ${ }^{(82-86)}$ foram publicados, desde a década de 90, utilizando a VNI para evitar a reintubação na falência respiratória pós-extubação, com resultados promissores. No entanto, dois estudos ${ }^{(87,88)}$ randomizados não confirmaram o benefício da VN1 como método de resgate da insuficiência respiratória após extubação. Avaliando os dois estudos, foi alocado um total de 302 pacientes não selecionados com falência respiratória identificada dentro de $48 \mathrm{~h}$ pós-extubação. Os pacientes foram randomizados em dois grupos; um grupo recebeu tratamento convencional (oxigênio, fisioterapia e broncodilatadores) e o outro recebeu VN1 por máscara facial associada ao tratamento convencional. Em ambos os estudos, não foram observadas, entre os dois grupos, diferenças significativas nas taxas de reintubação, no tempo de permanência e na mortalidade, tanto hospitalar quanto em UTI. Uma análise posterior, avaliando apenas os pacientes com hipercapnia (incluindo os portadores de DPOC), também não observou quaisquer diferenças. Baseando-se nesses dois estudos, pode-se concluir que a VNI não foi eficaz em evitar a reintubação na falência respiratória que ocorreu após a extubação em grupos de pacientes não selecionados.

Recomendação: Ventilação não invasiva na falência respiratória pós-extubação pode ter riscos e aumentar a mortalidade, principalmente se houver uma demora em se proceder à reintubação.

Grau de recomendação: A

Comentário: Um estudo multicêntrico randomizado, envolvendo 221 pacientes não selecionados com falência respiratória pós-extubação, observou uma maior mortalidade na UTI no grupo que recebeu VN1 em relação ao grupo que recebeu tratamento convencional $(25 \% \text { vs. } 14 \%, p=0,048)^{(88)}$. Um dado extremamente importante observado nesse estudo foi que o intervalo de tempo entre o desenvolvimento da falência respiratória e a reintubação foi significativamente maior no grupo que recebeu VN1 em relação ao grupo que recebeu tratamento convencional ( $12 \mathrm{~h}$ vs. $2 \mathrm{~h}$ e $30 \mathrm{~min}, \mathrm{p}=0,02$ ). Esse retardo na reintubação pode ter aumentado a mortalidade no grupo que recebeu VNI. A causa do aumento da mortalidade nesses casos se deveu a vários mecanismos tais como isquemia cardíaca, 
aumento da fadiga muscular respiratória, pneumonite por aspiração e complicações da intubação de emergência.

Área de controvérsia: Três estudos randomizados avaliaram o uso precoce e sistemático da VNI após a extubação como forma de prevenir a insuficiência respiratória pós-extubação, com resultados controversos. Jiang et al. ${ }^{(89)}$, avaliou 93 pacientes que foram extubados e imediatamente randomizados para receber VNI com PS + PEEP por máscara facial ou oxigenioterapia. Não foram observadas diferenças significativas na taxa de reintubação entre os grupos. Esse estudo apresentou problemas na randomização, pois a maioria dos pacientes com extubação não programada recebeu VNI, e esse foi o grupo que mais necessitou de reintubação. Nava e colaboradores avaliaram 97 pacientes em ventilação mecânica por mais de $48 \mathrm{~h}$ e considerados de alto risco para insuficiência respiratória pós-extubação (hipercapnia, insuficiência cardíaca congestiva, tosse ineficaz, secreção traqueobrônquica excessiva, mais de uma falência em tentativas de desmame, mais de uma comorbidade e obstrução de via aérea superior). Esses pacientes foram randomizados para receber VNI ou tratamento convencional após a extubação. Aqueles que receberam VNI tiveram menor taxa de reintubação $(8,3 \%$ vs. $24,5 \%)$ e menor mortalidade na UTI ${ }^{(90)}$. No estudo de Ferrer, 162 pacientes que toleraram o teste de respiração espontânea, mas considerados de alto risco para reintubação (idade > 65 anos, falência cardíaca como causa da intubação ou APACHE $11>12$ no dia da extubação), foram randomizados para $24 \mathrm{~h}$ de VNl com PS+PEEP ou oxigenoterapia, logo após a extubação. 0 desfecho primário foi redução da ocorrência de insuficiência respiratória pós-extubação. Esse desfecho foi menos freqüente no grupo que recebeu VNI (16\% vs 33\% no grupo oxigenioterapia). A mortalidade na UTI também foi menor nesse grupo, porém não houve diferença da mortalidade em 90 dias. Uma comparação entre pacientes com e sem hipercapnia durante o teste de ventilação espontânea sugere benefício maior da VN1 nos pacientes hipercápnicos ${ }^{(91)}$.

\section{Ventilação não-invasiva como estratégia de desmame}

Recomendação: A VN1 através de máscara facial como estratégia de desmame pode ser utilizada em paciente com repetidas falhas no teste de respiração espontânea, porém as evidências de seu benefício ainda são consideradas insuficientes.

Grau de recomendação: B

Comentário: Três grandes estudos randomizados abordaram o uso da ventilação não invasiva como técnica de desmame ${ }^{(92-94)}$. Nesses estudos, foram incluídos pacientes submetidos à ventilação mecânica por 24 a 48 h e que, após atingirem os critérios para desmame, falharam no teste de ventilação espontânea. Esses pacientes foram randomizados em duas estratégias de desmame: um grupo foi extubado, e imediatamente submetido à VNl por máscara facial e o outro grupo permaneceu intubado e submetido à ventilação com pressão de suporte em redução progressiva, ou à ventilação assistida-controlada associada a testes de respiração espontânea através de tubo $\mathrm{T}$ diariamente. Analisando os três estudos em conjunto, a duração média da VNI foi de 13 a $24 \mathrm{~h} /$ dia, nas 24 a $48 \mathrm{~h}$ iniciais, aumentando-se, progressivamente, os intervalos sem suporte ventilatório. A VNI foi aplicada através de máscara facial, com PS variando de 5 a $15 \mathrm{cmH}_{2} \mathrm{O}$ e PEEP de 4 a $5 \mathrm{cmH}_{2} \mathrm{O}$, objetivando FR $<25$ irpm, troca gasosa adequada e conforto do paciente. Em dois desses estudos ${ }^{(92,94)}$, o grupo submetido à VNl apresentou redução na duração da ventilação mecânica, na taxa de pneumonia e de choque séptico, proporcionando assim aumento na sobrevida em UTl e na sobrevida de 60 e 90 dias. A menor duração da ventilação mecânica observada no grupo que recebeu $\mathrm{VNl}$ ainda permitiu redução na permanência em UTl e hospitalar. Um dos estudos ${ }^{(93)}$ não demonstrou diferenças significativas entre os pacientes que receberam $\mathrm{VNI}$ e o grupo controle, respectivamente, nas taxas de sucesso do desmame, complicações, estadia na UTI, estadia hospitalar e sobrevida em 3 meses.

Uma metanálise recente ${ }^{(95)}$ selecionou cinco ensaios controlados e randomizados, considerados de moderada a boa qualidade, abordando o uso da VNI como estratégia de desmame para pacientes intubados e submetidos à ventilação mecânica para tratamento de insuficiência respiratória. Foi incluído um total de 171 pacientes, com predomínio de portadores de DPOC. A conclusão foi que a VN1 como estratégia de desmame reduziu a duração da intubação endotraqueal, a duração total do suporte ventilatório, a incidência de pneumonia associada ao ventilador $(R R=0,28,1 C 95 \%=0,09-0,85)$, a permanência na UT1 e hospitalar, e a mortalidade 


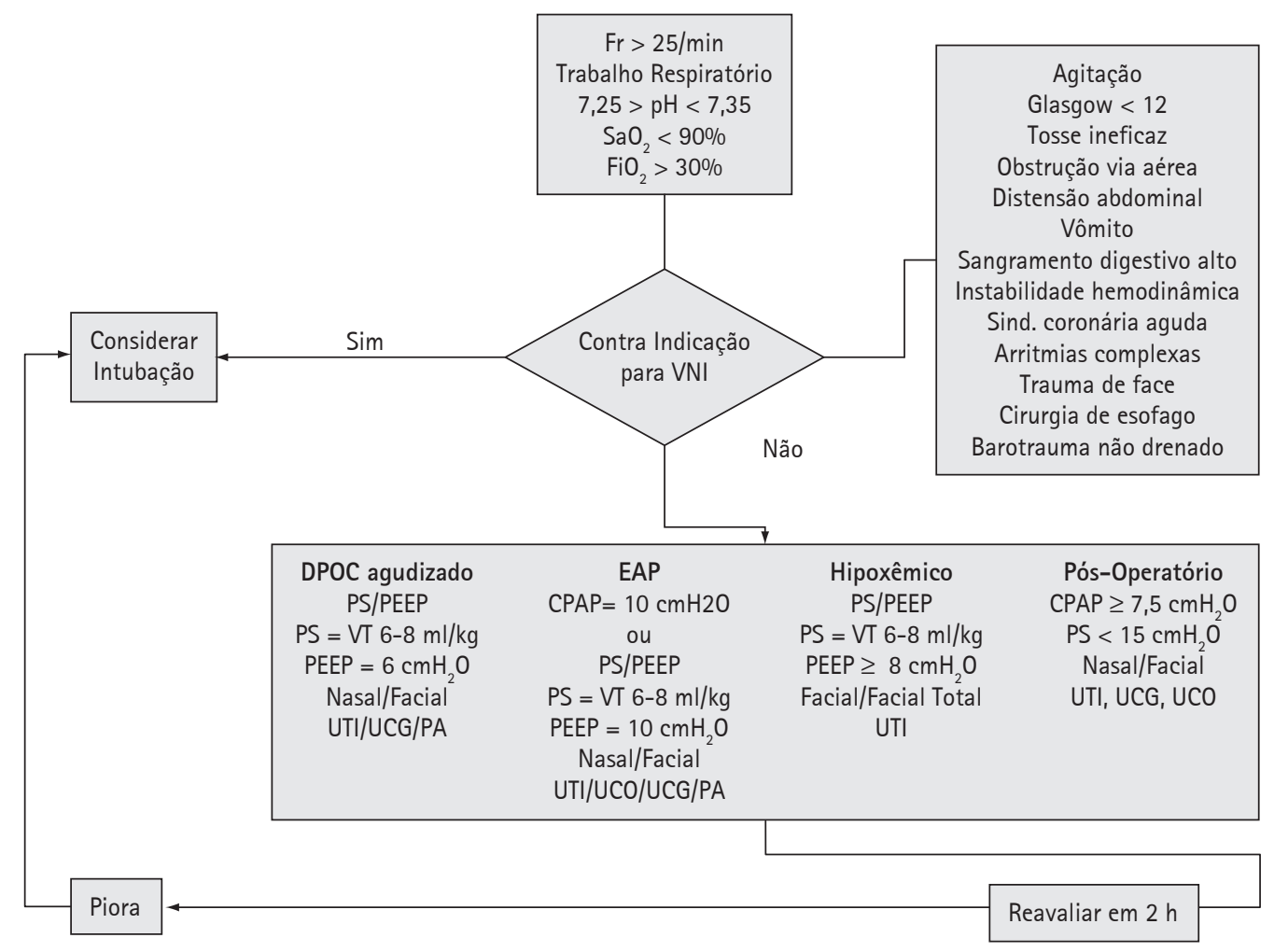

Figura 1 - Fluxograma.

$(R R=0,41, \quad 1 C 95 \%=0,22-0,76) . A$ análise do subgrupo de pacientes em ventilação mecânica por exacerbação de DPOC demonstrou que esses foram os que mais se beneficiaram do uso da $\mathrm{VNI}$ como estratégia de desmame. Ainda assim, a evidência foi considerada insuficiente para recomendar seu uso rotineiro.

Dessa forma, a VNI como estratégia de desmame foi considerada promissora, embora evidências do seu benefício clínico ainda sejam insuficientes.

\section{Referências}

1. International Consensus Conferences in Intensive Care Medicine: noninvasive positive pressure ventilation in acute Respiratory failure. Am J Respir Crit Care Med. 2001;163(1):283-91.

2. Wyatt J, Bellis F. British Thoracic Society guidelines on noninvasive ventilation. Emerg Med J. 2002;19(5):435.

3. Soo Hoo GW, Santiago S, Williams AJ. Nasal mechanical ventilation for hypercapnic respiratory failure in chronic obstructive pulmonary disease: determinants of success and failure. Crit Care Med. 1994;22(8):1253-61.

4. Richards GN, Cistulli PA, Ungar RG, Berthon-Jones M, Sullivan CE. Mouth leak with nasal continuous positive airway pressure increases nasal airway resistance. Am J Respir Crit Care Med. 1996;154(1):182-6.

5. Martins De Araujo MT, Vieira SB, Vasquez EC, Fleury B. Heated humidification or face mask to prevent upper airway dryness during continuous positive airway pressure therapy. Chest. 2000;117(1):142-7.

6. Navalesi P, Fanfulla F, Frigerio P, Gregoretti C, Nava S. Physiologic evaluation of noninvasive mechanical ventilation delivered with three types of masks in patients with chronic hypercapnic respiratory failure. Crit Care Med. 2000;28(6):1785-90.

7. Kwok H, McCormack J, Cece R, Houtchens J, Hill NS. Controlled trial of oronasal versus nasal mask ventilation in the treatment of acute respiratory failure. Crit Care Med. 2003;31(2):468-73.

8. Schettino GP, Chatmongkolchart S, Hess DR, Kacmarek RM. Position of exhalation port and mask design affect $\mathrm{CO} 2$ rebreathing during noninvasive positive pressure ventilation. Crit Care Med. 2003;31(8):2178-82.

9. Criner GJ, Travaline JM, Brennan KJ, Kreimer DT. Efficacy of a new full face mask for noninvasive positive pressure ventilation. Chest. 1994;106(4):1109-15.

10. Antonelli M, Pennisi MA, Pelosi P, Gregoretti C, Squadrone $\mathrm{V}$, Rocco $\mathrm{M}$ et al. Noninvasive positive pressure ventilation using a helmet in patients with acute exacerbation of chronic obstructive pulmonary disease: a feasibility study. Anesthesiology. 2004;100(1):16-24. 
11. Taccone P, Hess D, Caironi P, Bigatello LM. Continuous positive airway pressure delivered with a "helmet": effects on carbon dioxide rebreathing. Crit Care Med. 2004;32(10):2090-6.

12. Ferguson GT, Gilmartin M. CO2 rebreathing during BiPAP ventilatory assistance. Am J Respir Crit Care Med. 1995;151(4):1126-35.

13. Lofaso F, Brochard L, Hang T, Lorino H, Harf A, Isabey D. Home versus intensive care pressure support devices. Experimental and clinical comparison. Am J Respir Crit Care Med. 1996;153(5):1591-9.

14. Bunburaphong T, Imanaka H, Nishimura M, Hess D, Kacmarek RM. Performance characteristics of bilevel pressure ventilators: a lung model study. Chest. 1997;111(4):1050-60.

15. Stell IM, Paul G, Lee KC, Ponte J, Moxham J. Noninvasive ventilator triggering in chronic obstructive pulmonary disease. A test lung comparison. Am J Respir Crit Care Med. 2001;164(11):2092-7.

16. Vitacca M, Barbano L, D’Anna S, Porta R, Bianchi L, Ambrosino N. Comparison of five bilevel pressure ventilators in patients with chronic ventilatory failure: a physiologic study. Chest. 2002;122(6):2105-14.

17. Hess DR. The evidence for noninvasive positive-pressure ventilation in the care of patients in acute respiratory failure: a systematic review of the literature. Respir Care. 2004;49(7):810-29.

18. Porta R, Appendini L, Vitacca M, Bianchi L, Donner CF, Poggi R et al. Mask proportional assist vs pressure support ventilation in patients in clinically stable condition with chronic ventilatory failure. Chest. 2002;122(2):479-88.

19. Vitacca M, Clini E, Pagani M, Bianchi L, Rossi A, Ambrosino N. Physiologic effects of early administered mask proportional assist ventilation in patients with chronic obstructive pulmonary disease and acute respiratory failure. Crit Care Med. 2000;28(6):1791-7.

20. Wysocki M, Richard JC, Meshaka P. Noninvasive proportional assist ventilation compared with noninvasive pressure support ventilation in hypercapnic acute respiratory failure. Crit Care Med. 2002;30(2):323-9.

21. Masip J, Betbese AJ, Paez J, Vecilla F, Canizares R, Padro J, et al. Non-invasive pressure support ventilation versus conventional oxygen therapy in acute cardiogenic pulmonary oedema: a randomised trial. Lancet. 2000;356(9248):2126-32.

22. Park M, Sangean MC, Volpe Mde S, Feltrim Ml, Nozawa E, Leite PF, et al. Randomized, prospective trial of oxygen, continuous positive airway pressure, and bilevel positive airway pressure by face mask in acute cardiogenic pulmonary edema. Crit Care Med. 2004;32(12):2407-15.

23. Fu C, Caruso P, Lucatto JJ, de Paula Schettino GP, de Souza R, Carvalho CR. Comparison of two flow generators with a noninvasive ventilator to deliver continuous positive airway pressure: a test lung study. Intensive Care Med. 2005;31(11):1587-91.

24. Lightowler JV, Wedzicha JA, Elliott MW, Ram FS. Noninvasive positive pressure ventilation to treat respiratory failure resulting from exacerbations of chronic obstructive pulmonary disease: Cochrane systematic review and metaanalysis. Bmj. 2003;326(7382):185.

25. Keenan SP, Sinuff T, Cook DJ, Hill NS. Which patients with acute exacerbation of chronic obstructive pulmonary disease benefit from noninvasive positive-pressure ventilation? A systematic review of the literature. Ann Intern Med. 2003;138(11):861-70.
26. Ram FS, Picot J, Lightowler J, Wedzicha JA. Non-invasive positive pressure ventilation for treatment of respiratory failure due to exacerbations of chronic obstructive pulmonary disease. Cochrane Database Syst Rev. 2004(3):CD004104.

27. Bott J, Carroll MP, Conway JH, Keilty SE, Ward EM, Brown $\mathrm{AM}$, et al. Randomised controlled trial of nasal ventilation in acute ventilatory failure due to chronic obstructive airways disease. Lancet. 1993;341(8860):1555-7.

28. Brochard L, Mancebo J, Wysocki M, Lofaso F, Conti G, Rauss $A$, et al. Noninvasive ventilation for acute exacerbations of chronic obstructive pulmonary disease. $\mathrm{N}$ Engl J Med. 1995;333(13):817-22.

29. Angus RM, Ahmed AA, Fenwick LJ, Peacock AJ. Comparison of the acute effects on gas exchange of nasal ventilation and doxapram in exacerbations of chronic obstructive pulmonary disease. Thorax. 1996;51(10):1048-50.

30. Barbe F, Togores B, Rubi M, Pons S, Maimo A, Agusti AG. Noninvasive ventilatory support does not facilitate recovery from acute respiratory failure in chronic obstructive pulmonary disease. Eur Respir J. 1996;9(6):1240-5.

31. Celikel T, Sungur M, Ceyhan B, Karakurt S. Comparison of noninvasive positive pressure ventilation with standard medical therapy in hypercapnic acute respiratory failure. Chest. 1998;114(6):1636-42.

32. Dikensoy 0, lkidag B, Filiz A, Bayram N. Comparison of noninvasive ventilation and standard medical therapy in acute hypercapnic respiratory failure: a randomised controlled study at a tertiary health centre in SE Turkey. Int J Clin Pract. 2002;56(2):85-8.

33. Plant PK, Owen JL, Elliott MW. Early use of noninvasive ventilation for acute exacerbations of chronic obstructive pulmonary disease on general respiratory wards: a multicentre randomised controlled trial. Lancet. 2000;355(9219):1931-5.

34. Bardi G, Pierotello R, Desideri M, Valdisserri L, Bottai M, Palla A. Nasal ventilation in COPD exacerbations: early and late results of a prospective, controlled study. Eur Respir J. 2000;15(1):98-104.

35. Collaborative Research Group of Noninvasive Mechanical Ventilation for Chronic Obstructive Pulmonary Disease. Early use of non-invasive positive pressure ventilation for acute exacerbations of chronic obstructive pulmonary disease: a multicentre randomized controlled trial. Chin Med J (Engl). 2005;118(24):2034-40.

36. Meduri GU, Cook TR, Turner RE, Cohen M, Leeper KV. Noninvasive positive pressure ventilation in status asthmaticus. Chest. 1996;110(3):767-74.

37. Soroksky A, Stav D, Shpirer 1. A pilot prospective, randomized, placebo-controlled trial of bilevel positive airway pressure in acute asthmatic attack. Chest. 2003;123(4):1018-25.

38. Ram FS, Wellington S, Rowe B, Wedzicha JA. Non-invasive positive pressure ventilation for treatment of respiratory failure due to severe acute exacerbations of asthma. Cochrane Database Syst Rev. 2005(3):CD004360.

39. Rasanen J, Heikkila J, Downs J, Nikki P, Vaisanen I, Viitanen A. Continuous positive airway pressure by face mask in acute cardiogenic pulmonary edema. Am J Cardiol. 1985;55(4):296-300.

40. Bersten AD, Holt AW, Vedig AE, Skowronski GA, Baggoley $C J$. Treatment of severe cardiogenic pulmonary edema with continuous positive airway pressure delivered by face mask. N Engl J Med. 1991;325(26):1825-30. 
41. Lin M, Yang YF, Chiang HT, Chang MS, Chiang BN, Cheitlin MD. Reappraisal of continuous positive airway pressure therapy in acute cardiogenic pulmonary edema. Short-term results and long-term follow-up. Chest. 1995;107(5):1379-86.

42. Pang D, Keenan SP, Cook DJ, Sibbald WJ. The effect of positive pressure airway support on mortality and the need for intubation in cardiogenic pulmonary edema: a systematic review. Chest. 1998;114(4):1185-92.

43. L'Her E, Duquesne F, Girou E, de Rosiere XD, Le Conte P, Renault $\mathrm{S}$, et al. Noninvasive continuous positive airway pressure in elderly cardiogenic pulmonary edema patients. Intensive Care Med. 2004;30(5):882-8.

44. Masip J, Roque M, Sanchez B, Fernandez R, Subirana M, Exposito JA. Noninvasive ventilation in acute cardiogenic pulmonary edema: systematic review and meta-analysis. JAMA. 2005;294(24):3124-30.

45. Park M, Lorenzi-Filho G. Noninvasive mechanical ventilation in the treatment of acute cardiogenic pulmonary edema. Clinics. 2006;61(3):247-52.

46. Mehta S, Jay GD, Woolard RH, Hipona RA, Connolly EM, Cimini DM, et al. Randomized, prospective trial of bilevel versus continuous positive airway pressure in acute pulmonary edema. Crit Care Med. 1997;25(4):620-8.

47. Bellone A, Vettorello M, Monari A, Cortellaro F, Coen D. Noninvasive pressure support ventilation vs. continuous positive airway pressure in acute hypercapnic pulmonary edema. Intens Care Med. 2005;31(6):807-11.

48. Nava S, Carbone G, DiBattista N, Bellone A, Baiardi P, Cosentini $R$ et al. Noninvasive ventilation in cardiogenic pulmonary edema: a multicenter randomized trial. Am J Respir Crit Care Med. 2003;168(12):1432-7.

49. Crane SD, Elliott MW, Gilligan P, Richards K, Gray AJ. Randomised controlled comparison of continuous positive airways pressure, bilevel non-invasive ventilation, and standard treatment in emergency department patients with acute cardiogenic pulmonary oedema. Emerg Med J. 2004;21(2):155-61.

50. Evans TW. International Consensus Conferences in Intensive Care Medicine: non-invasive positive pressure ventilation in acute respiratory failure.Organised jointly by the American Thoracic Society, the European Respiratory Society, the European Society of Intensive Care Medicine, and the Societe de Reanimation de Langue Francaise, and approved by the ATS Board of Directors, December 2000. Intens Care Med. 2001;27(1):166-78.

51. Non-invasive ventilation in acute respiratory failure. Thorax 2002;57(3):192-211.

52. Liesching T, Kwok H, Hill NS. Acute applications of noninvasive positive pressure ventilation. Chest 2003;124(2):699-713.

53. Mehta S, Hill NS. Noninvasive ventilation. Am J Respir Crit Care Med. 2001;163(2):540-77.

54. Antonelli M, Conti G, Rocco M, Bufi M, De Blasi RA, Vivino $\mathrm{G}$, et al. A comparison of noninvasive positive-pressure ventilation and conventional mechanical ventilation in patients with acute respiratory failure. $\mathrm{N}$ Engl $\mathrm{J}$ Med. 1998;339(7):429-35

55. Ferrer M, Esquinas A, Leon M, Gonzalez G, Alarcon A, Torres A. Noninvasive ventilation in severe hypoxemic respiratory failure: a randomized clinical trial. Am J Respir Crit Care Med. 2003;168(12):1438-44.

56. Keenan SP, Kernerman PD, Cook DJ, Martin CM, McCormack D, Sibbald WJ. Effect of noninvasive positive pressure ventilation on mortality in patients admitted with acute respiratory failure: a meta-analysis. Crit Care Med. 1997;25(10):1685-92.

57. Peter JV, Moran JL, Phillips-Hughes J, Warn D. Noninvasive ventilation in acute respiratory failure--a meta-analysis update. Crit Care Med. 2002;30(3):555-62.

58. Keenan SP, Sinuff T, Cook DJ, Hill NS. Does noninvasive positive pressure ventilation improve outcome in acute hypoxemic respiratory failure? A systematic review. Crit Care Med. 2004;32(12):2516-23.

59. Antonelli M, Conti G, Bufi M, Costa MG, Lappa A, Rocco M, et al. Noninvasiveventilation for treatment of acute respiratory failure in patients undergoing solid organ transplantation: a randomized trial. JAMA. 2000;283(2):235-41.

60. Hilbert G, Gruson D, Vargas F, Valentino R, GbikpiBennisan G, Dupon M, et al. Noninvasive ventilation in immunosuppressed patients with pulmonary infiltrates, fever, and acute respiratory failure. $\mathrm{N}$ Engl J Med. 2001;344(7):481-7.

61. Confalonieri M, Calderini E, Terraciano S, Chidini G, Celeste E, Puccio G, et al. Noninvasive ventilation for treating acute respiratory failure in AIDS patients with Pneumocystis carinii pneumonia. Intens Care Med. 2002;28(9):1233-8.

62. Confalonieri M, Potena A, Carbone G, Porta RD, Tolley EA, Umberto Meduri G. Acute respiratory failure in patients with severe community-acquired pneumonia. A prospective randomized evaluation of noninvasive ventilation. Am J Respir Crit Care Med. 1999;160(5 Pt 1):1585-91.

63. Auriant 1 , Jallot A, Herve P, Cerrina J, Le Roy Ladurie F, Fournier JL, et al. Noninvasive ventilation reduces mortality in acute respiratory failure following lung resection. Am J Respir Crit Care Med. 2001;164(7):1231-5.

64. Rocker GM, Mackenzie MG, Williams B, Logan PM. Noninvasive positive pressure ventilation: successful outcome in patients with acute lung injury/ARDS. Chest. 1999;115(1):173-7.

65. Meduri GU, Fox RC, Abou-Shala N, Leeper KV, Wunderink RG. Noninvasive mechanical ventilation via face mask in patients with acute respiratory failure who refused endotracheal intubation. Crit Care Med. 1994;22(10):1584-90.

66. Benhamou D, Girault C, Faure C, Portier F, Muir JF. Nasal mask ventilation in acute respiratory failure. Experience in elderly patients. Chest. 1992;102(3):912-7.

67. Chu CM, Chan VL, Wong IW, Leung WS, Lin AW, Cheung KF. Noninvasive ventilation in patients with acute hypercapnic exacerbation of chronic obstructive pulmonary disease who refused endotracheal intubation. Crit Care Med. 2004;32(2):372-7.

68. Levy M, Tanios MA, Nelson D, Short K, Senechia A, Vespia $\mathrm{J}$, et al. Outcomes of patients with do-not-intubate orders treated with noninvasive ventilation. Crit Care Med 2004;32(10):2002-7.

69. Schettino G, Altobelli N, Kacmarek RM. Noninvasive positive pressure ventilation reverses acute respiratory failure in select "do-not-intubate" patients. Crit Care Med. 2005;33(9):1976-82.

70. Ricksten SE, Bengtsson A, Soderberg C, Thorden M, Kvist H. Effects of periodic positive airway pressure by mask on postoperative pulmonary function. Chest. 1986;89(6):774-81.

71. Celli BR, Rodriguez KS, Snider GL. A controlled trial of intermittent positive pressure breathing, incentive spirometry, and deep breathing exercises in preventing pulmonary 
complications after abdominal surgery. Am Rev Respir Dis. 1984;130(1):12-5.

72. Schuppisser JP, Brandli 0, Meili U. Postoperative intermittent positive pressure breathing versus physiotherapy. Am J Surg. 1980;140(5):682-6.

73. Kindgen-Milles D, Buhl R, Gabriel A, Bohner H, Muller E. Nasal continuous positive airway pressure: A method to avoid endotracheal reintubation in postoperative high-risk patients with severe nonhypercapnic oxygenation failure. Chest. 2000;117(4):1106-11.

74. Varon J, Walsh GL, Fromm RE, Jr. Feasibility of noninvasive mechanical ventilation in the treatment of acute respiratory failure in postoperative cancer patients. J Crit Care. 1998;13(2):55-7.

75. Ebeo CT, Benotti PN, Byrd RP, Jr., Elmaghraby Z, Lui J. The effect of bi-level positive airway pressure on postoperative pulmonary function following gastric surgery for obesity. Respir Med. 2002;96(9):672-6.

76. Joris JL, Sottiaux TM, Chiche JD, Desaive CJ, Lamy ML. Effect of bi-level positive airway pressure (BiPAP) nasal ventilation on the postoperative pulmonary restrictive syndrome in obese patients undergoing gastroplasty. Chest. 997;111(3):665-70.

77. Squadrone V, Coha M, Cerutti E, Schellino MM, Biolino $\mathrm{P}$, Occella $\mathrm{P}$, et al. Continuous positive airway pressure for treatment of postoperative hypoxemia: a randomized controlled trial. JAMA. 2005;293(5):589-95.

78. Aguilo R, Togores B, Pons S, Rubi M, Barbe F, Agusti AG. Noninvasive ventilatory support after lung resectional surgery. Chest. 1997;112(1):117-21.

79. Gust R, Gottschalk A, Schmidt H, Bottiger BW, Bohrer H, Martin E. Effects of continuous (CPAP) and bi-level positive airway pressure (BiPAP) on extravascular lung water after extubation of the trachea in patients following coronary artery bypass grafting. Intensive Care Med. 1996;22(12):1345-50.

80. Matte P, Jacquet L, Van Dyck M, Goenen M. Effects of conventional physiotherapy, continuous positive airway pressure and non-invasive ventilatory support with bilevel positive airway pressure after coronary artery bypass grafting. Acta Anaesthesiol Scand. 2000;44(1):75-81.

81. Pasquina P, Merlani P, Granier JM, Ricou B. Continuous positive airway pressure versus noninvasive pressure support ventilation to treat atelectasis after cardiac surgery. Anesth Analg. 2004;99(4):1001-8, table of contents.

82. Meduri GU, Conoscenti CC, Menashe P, Nair S. Noninvasive face mask ventilation in patients with acute respiratory failure. Chest. 1989;95(4):865-70.

83. Hilbert G, Gruson D, Portel L, Gbikpi-Benissan G, Cardinaud JP. Noninvasive pressure support ventilation in COPD patients with postextubation hypercapnic respiratory insufficiency. Eur Respir J. 1998;11(6):1349-53.
84. Munshi IA, DeHaven B, Kirton O, Sleeman D, Navarro M. Reengineering respiratory support following extubation: avoidance of critical care unit costs. Chest. 1999;116(4):1025-8.

85. Kilger E, Briegel J, Haller M, Frey L, Schelling G, Stoll C, et al. Effects of noninvasive positive pressure ventilatory support in non-COPD patients with acute respiratory insufficiency after early extubation. Intens Care Med. 1999;25(12):1374-80.

86. Vitacca M, Ambrosino N, Clini E, Porta R, Rampulla C, Lanini B, et al. Physiological response to pressure support ventilation delivered before and after extubation in patients not capable of totally spontaneous autonomous breathing. Am J Respir Crit Care Med. 2001;164(4):638-41.

87. Keenan SP, Powers C, McCormack DG, Block G. Noninvasive positive-pressure ventilation for postextubation respiratory distress: a randomized controlled trial. JAMA. 2002;287(24):3238-44.

88. Esteban A, Frutos-Vivar F, Ferguson ND, Arabi Y, Apezteguia C, Gonzalez M, et al. Noninvasive positive-pressure ventilation for respiratory failure after extubation. $\mathrm{N}$ Engl J Med. 2004;350(24):2452-60.

89. Jiang JS, Kao SJ, Wang SN. Effect of early application of biphasic positive airway pressure on the outcome of extubation in ventilator weaning. Respirology. 1999;4(2):161-5.

90. Nava S, Gregoretti C, Fanfulla F, Squadrone E, Grassi M, Carlucci A, et al. Noninvasive ventilation to prevent respiratory failure after extubation in high-risk patients. Crit Care Med. 2005;33(11):2465-70.

91. Ferrer M, Valencia M, Nicolas JM, Bernadich 0, Badia JR, Torres A. Early noninvasive ventilation averts extubation failure in patients at risk: a randomized trial. Am J Respir Crit Care Med. 2006;173(2):164-70.

92. Nava S, Ambrosino N, Clini E, Prato M, Orlando G, Vitacca $\mathrm{M}$, et al. Noninvasive mechanical ventilation in the weaning of patients with respiratory failure due to chronic obstructive pulmonary disease. A randomized, controlled trial. Ann Intern Med. 1998;128(9):721-8.

93. Girault C, Daudenthun I, Chevron V, Tamion F, Leroy J, Bonmarchand G. Noninvasive ventilation as a systematic extubation and weaning technique in acute-on-chronic respiratory failure: a prospective, randomized controlled study. Am J Respir Crit Care Med. 1999;160(1):86-92.

94. Ferrer M, Esquinas A, Arancibia F, Bauer TT, Gonzalez G, Carrillo A, et al. Noninvasive ventilation during persistent weaning failure: a randomized controlled trial. Am J Respir Crit Care Med. 2003;168(1):70-6.

95. Burns KE, Adhikari NK, Meade MO. Noninvasive positive pressure ventilation as a weaning strategy for intubated adults with respiratory failure. Cochrane Database Syst Rev. 2003(4):CD004127. 